\title{
Par-delà le trauma
}

11 mars 2011, Iwate (Japon)

Beyond trauma 2011/03/11

\section{Shoichiro Takezawa}

\section{CpenEdition}

Journals

Édition électronique

URL : https://journals.openedition.org/tc/7789

DOI : $10.4000 /$ tc. 7789

ISSN : 1952-420X

Éditeur

Éditions de l'EHESS

\section{Édition imprimée}

Date de publication : 31 octobre 2016

Pagination : 48-59

ISBN : 9782713225291

ISSN : 0248-6016

\section{Référence électronique}

Shoichiro Takezawa, «Par-delà le trauma », Techniques \& Culture [En ligne], 65-66 | 2016, mis en ligne le 31 octobre 2018 , consulté le 29 septembre 2022. URL : http://journals.openedition.org/tc/7789 ; DOI : https://doi.org/10.4000/tc.7789 


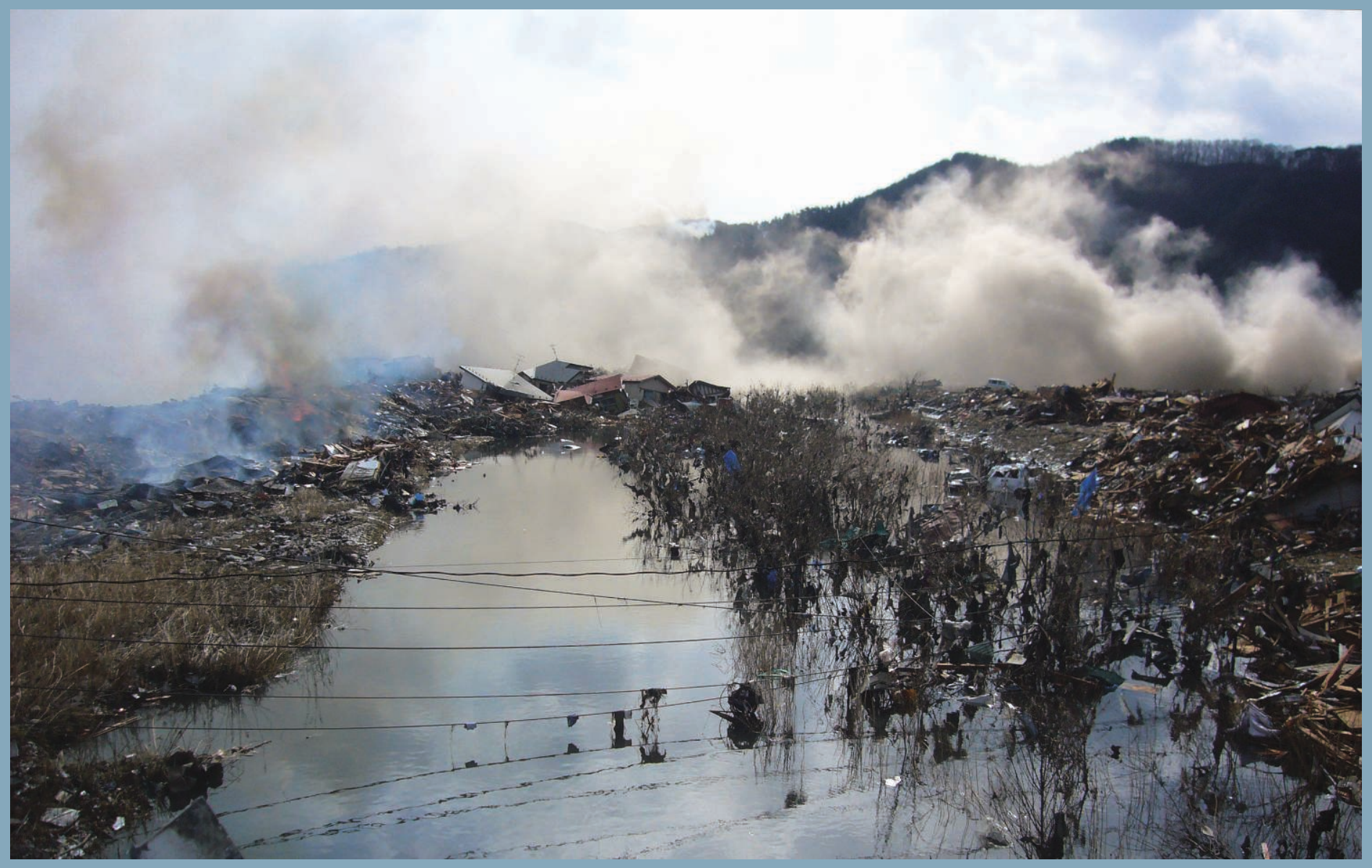




\section{Par-delà le trauma}

\section{1 mars 2011, Iwate (Japon)}

Par-delà le trauma, comment inventorier et conserver les restes des bâtiments détruits par le séisme Tohoku et comment réaliser une exposition sur cette catastrophe? Voici les questions simples mais fondamentales auxquelles je m’attacherai à répondre dans cet article.

\section{Une ville totalement détruite}

Le 11 mars 2011, un grand séisme a impacté le Nord-Est du Japon. D’une magnitude de 9,0, il est le plus intense jamais enregistré dans l'histoire du pays et l'un des plus grands que le monde ait connu. Pourtant ce n'est pas le tremblement de terre, mais bien le tsunami, d'une hauteur de 10 à 15 mètres, attaquant les régions côtières 40 minutes après, qui a provoqué d'immenses dégâts. En effet, 18455 personnes ont été tuées ou ont été portées disparues, 380000 personnes ont perdu leur maison et on estime à 2 billions de yen (150 milliards d'euros) la perte économique.

J'habite à Kyoto, à 1000 kilomètres des régions attaquées par le tsunami. J'ai ressenti un grand tremblement de terre durant environ 2 minutes. Constatant la corrélation de la durée et de l'intensité, notre inquiétude était d'autant plus grande. Nous, ma femme et moi, avons allumé la télévision qui n’a retransmis aucune image pendant des dizaines de minutes. Et une demi-heure plus tard, le cauchemar a recommencé et a pénétré jusqu'au fond de nos coeurs: la mer remontante a envahi la plaine et la ville, elle a entièrement dévasté toutes les maisons construites en bois. Chaque minute, chaque heure, de nouvelles images de plus en plus terrifiantes nous parvenaient. À force de voir, à la télévision, de nombreuses personnes qui avaient tout perdu, nous étions tout à la fois déchirés et interdits, ces sentiments ont perduré une quinzaine de jours. 
La ville d'Otsuchi avant la catastrophe

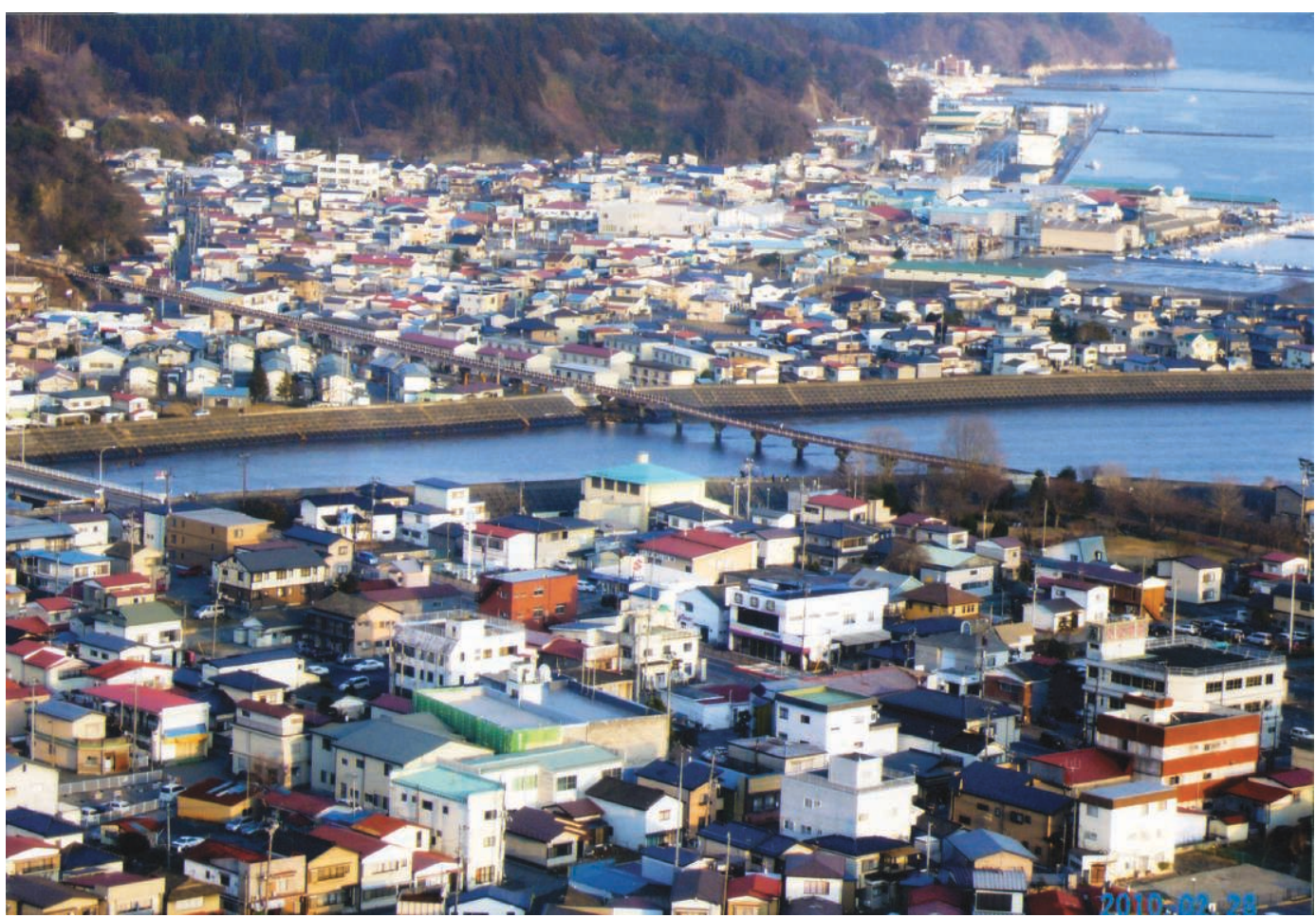

Nous avons alors discuté et décidé de nous rendre sur les lieux sinistrés, avec notre voiture, en tant que volontaires, pour soutenir les habitants. Parallèlement, l'Académie japonaise a envoyé, à tous les chercheurs, un message qui recommandait d'éviter de faire des recherches sur le terrain, et notamment de ne pas faire d'enquêtes chez les victimes afin d'éviter la surcharge de ces zones. C'est ainsi qu'agir en tant que volontaires devint la seule possibilité pour nous d'y aller.

Nous voulions aller dans une municipalité où il y avait peu de bénévoles. Le tsunami avait attaqué les trois municipalités suivantes, du nord au sud: Iwate, Miyagi et Fukushima. La préfecture de Miyagi ayant assez de bénévoles et comme nous pensions être inutiles dans la préfecture de Fukushima, nous avons décidé d'aller à Iwate. Nous avons choisi la ville d'Otsuchi pour travailler comme volontaires, car elle était la seule commune à accueillir des bénévoles parmi toutes les autres d'Iwate. Otsuchi (16000 habitants avant le tsunami) a été la ville la plus endommagée. En effet, $10 \%$ de la population a succombé et $60 \%$ des habitants ont perdu leur logement. Les principales activités de la ville provenaient de la pêche, de l'alimentation issue de produits de la mer, et de l'agriculture, mais le tsunami a tout anéanti.

Les photographies de la ville avant et après le séisme montrent le centre-ville ainsi que plusieurs communes qui se trouvent au bord de la mer complètement détruits. Les dégâts provoqués par le tsunami ont été aggravés par l'incendie qui a surgi juste après et qui a empêché l'accès 


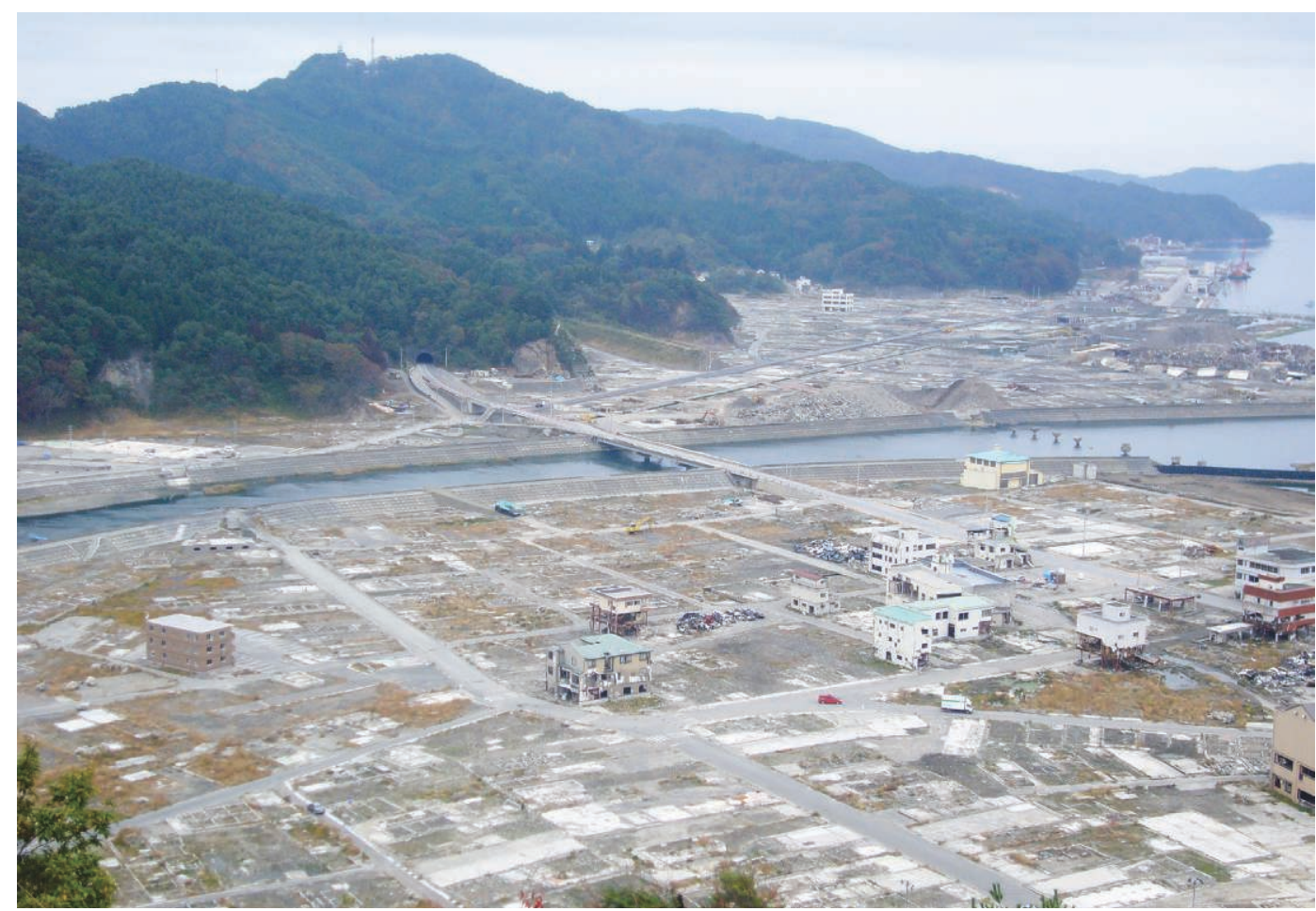

à la ville pour les secours extérieurs, y compris pour l'Armée. De ce fait, les journaux nationaux ont annoncé pendant 5 jours qu'il n'y avait aucune nouvelle des 10000 habitants d'Otsuchi.

Les photographies révèlent les structures principales de la ville: la mairie détruite et recouverte par les décombres des bâtiments, la caserne des sapeurs-pompiers et les digues de 6 mètres démolies par le tsunami. Plus dramatique: le maire et l'ensemble des responsables de la mairie sont morts (au total 40 personnes parmi 140 employés ont disparu). Durant trois semaines, la mairie n'a pu fonctionner; les activités d'entraide étaient le seul moyen de survie des administrés, en particulier pour les réfugiés qui avaient tout perdu.

Présentons maintenant brièvement ce que nous avons fait dans cette région dévastée. En tant que bénévoles, nous avons d'abord nettoyé et réparé des documents apportés par les officiers des forces d'autodéfenses: toutes sortes d'attestations, livrets de banque, titres de propriété foncière ainsi que des albums-photo. Quelques mois plus tard, nous avons commencé à donner des conseils à la population locale qui s'est engagée dans le planning urbain pour la reconstruction de la commune -j'avais moi-même des expériences de ce genre au sein d'autres communautés. Dans le même temps, nous avons réalisé des entretiens (environ 200 enregistrements) avec la population locale, à la fois comme bénévoles et comme chercheurs. De fait, nous n'avons jamais été sur place comme de simples observateurs.
2. La ville d'Otsuchi après la catastrophe 
3. La mairie d'Otsuchi complètement détruite par le tsunami, 40 employés en fonction y ont été tués.

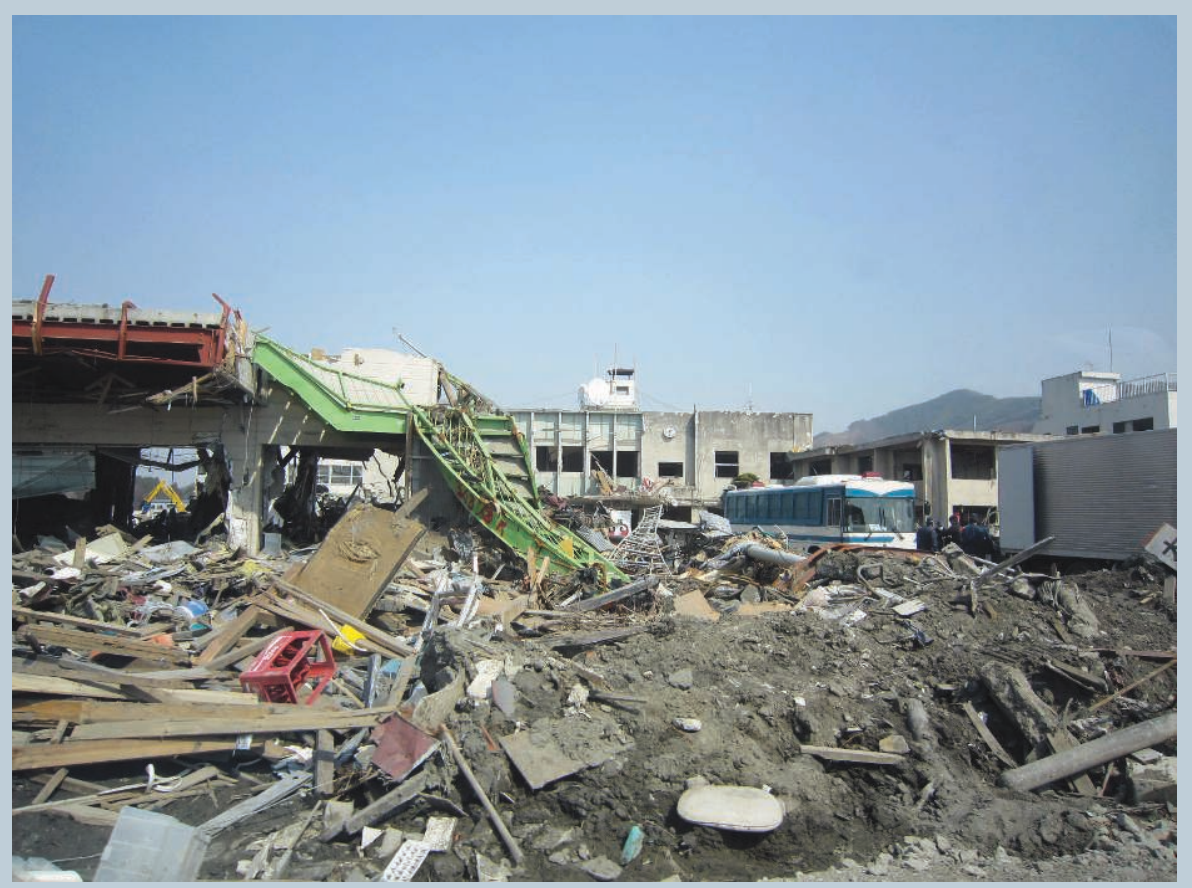

4. Beaucoup de photographies témoignant de la vie heureuse ont été retrouvées dans les décombres des maisons détruites par le tsunami.

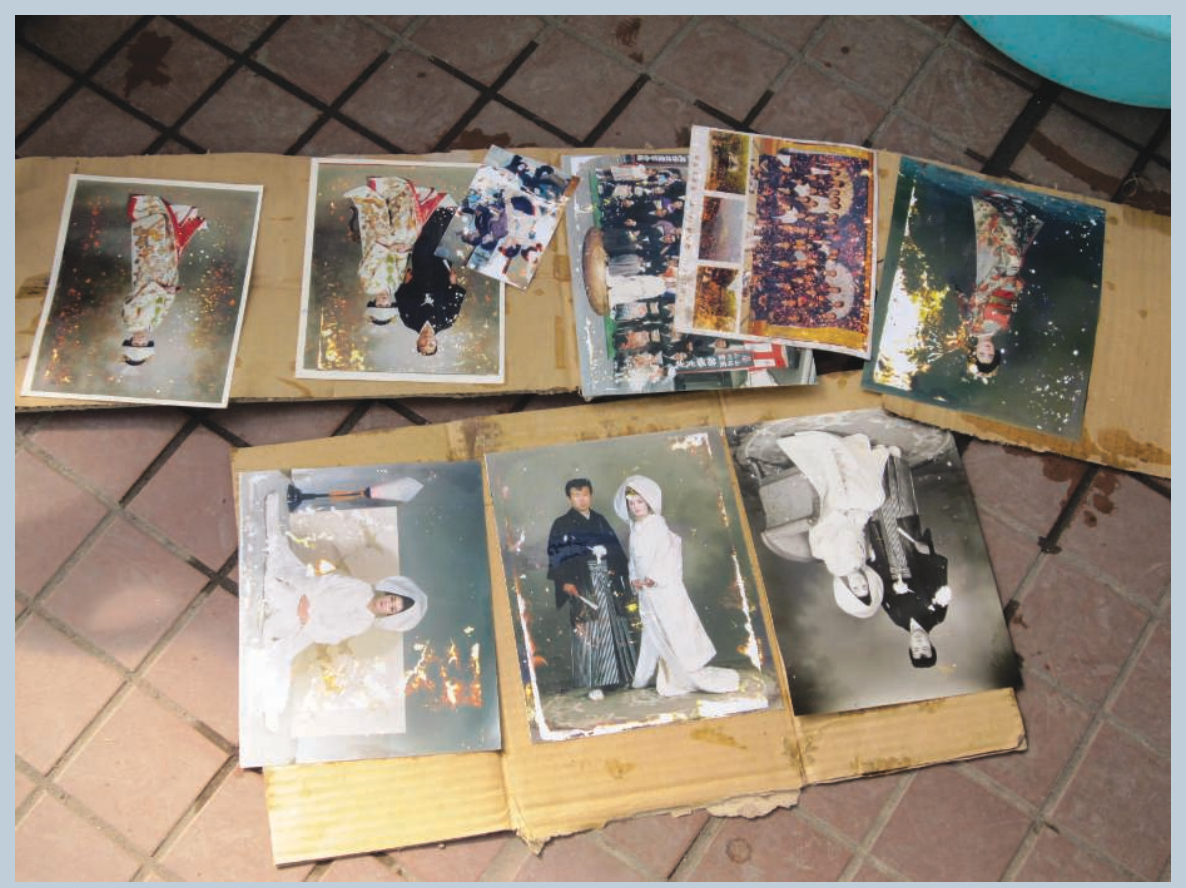




\section{Réaliser une exposition sur le tsunami}

Notre objectif fut ensuite de réaliser une exposition sur la catastrophe en nous concentrant sur la ville d'Otsuchi et de participer à la conservation des ruines de la mairie où près d'un tiers des employés avait péri par le tsunami. Pourquoi vouloir faire ces deux choses en même temps? Avant de l'expliquer, je voudrais revenir sur notre expérience personnelle.

Au cours de notre séjour dans la préfecture d'Iwate, nous avons travaillé avec plusieurs personnes parmi lesquelles une dame qui nous a raconté sa propre histoire sans que nous l'ayons sollicitée. Elle avait deux filles dont l'aînée l'avait quittée pour se marier avec quelqu'un de Kanto, près de Tokyo. La cadette, décidant de rester auprès de ses parents, travaillait à la mairie d'Otsuchi, s'était mariée et avait eu une fille. Elle a été tuée par le tsunami dans la mairie où elle était restée après le tremblement de terre. Quand son corps a été retrouvé et les funérailles terminées, la dame a dit à son gendre de rompre les relations avec elle, car son épouse n'existait plus, et de retourner avec sa propre fille dans sa ville natale pour y travailler. Cette dame a donc perdu sa fille et sa petite fille dans la catastrophe. Elle n'a jamais pleuré devant nous, mais nous a raconté son histoire avec un visage empli de chagrin que nous ne pouvons pas oublier. Elle a répété: «Pourquoi n’ai-je pas été tuée?» «Pourquoi moi seule ai-je échappé à la mort?». Quand aujourd'hui je me rappelle son visage, la tristesse m'envahit jusqu'au fond du cœur et je ne peux rien faire pendant un certain temps. Je dois être frappé par une sorte de traumatisme. Les gens étrangers à la catastrophe, comme moi, peuvent être saisis d'un trauma; tous les habitants d'Otsuchi sont plus encore les victimes d'un choc traumatique important, qui persiste encore aujourd'hui. Ils ont perdu des membres de leur famille, leurs parents, des amis et des voisins.

Je prépare actuellement une exposition au musée d'ethnologie d'Osaka où je travaille et envisage ensuite de la transporter à Otsuchi afin de la présenter pendant une longue période. Dans un tel contexte, réaliser une exposition sur la catastrophe n'est pas un travail aisé car cela risque de provoquer ou de faire ressurgir le traumatisme chez ses habitants.

Pour montrer combien c'est une tâche difficile, je prendrai l'exemple suivant. Le séisme de Tohoku a détruit toutes les régions côtières et laissé plusieurs ruines: un bateau de mer emporté par le tsunami gît sur un bâtiment privé, un grand bateau de pêche a été repoussé à 500 mètres de la côte, un bâtiment de prévention des sinistres a été anéanti provoquant la mort de quelques dizaines de personnes, les étages inférieurs d'un hôtel privé ont été complètement dévastés. Tous ces restes ont déjà été démolis ou prévu de l'être, à l'exception de cet hôtel car personne n'y a été tué. Il est donc devenu un symbole de coopération, d'entraide et de bienveillance que les gens souhaitent conserver. Par contre, les autres ruines, où beaucoup de gens ont perdu la vie, ne représentent pour les habitants que le signe de la misère et de l'impuissance humaine.

Continuons d'inventorier ces ruines: un centre de gymnastique où plus de cent personnes ont été tuées (déjà démoli), un bâtiment de refuge dans lequel plus de cent réfugiés sont morts (sa destruction a été décidée) et une école d'Okawa dont 87 élèves ont péri (la démolition est en cours de discussion mais un procès est déjà instruit). 
5. Un grand bateau touristique a été emporté par le tsunami et a échoué sur une maison privée.

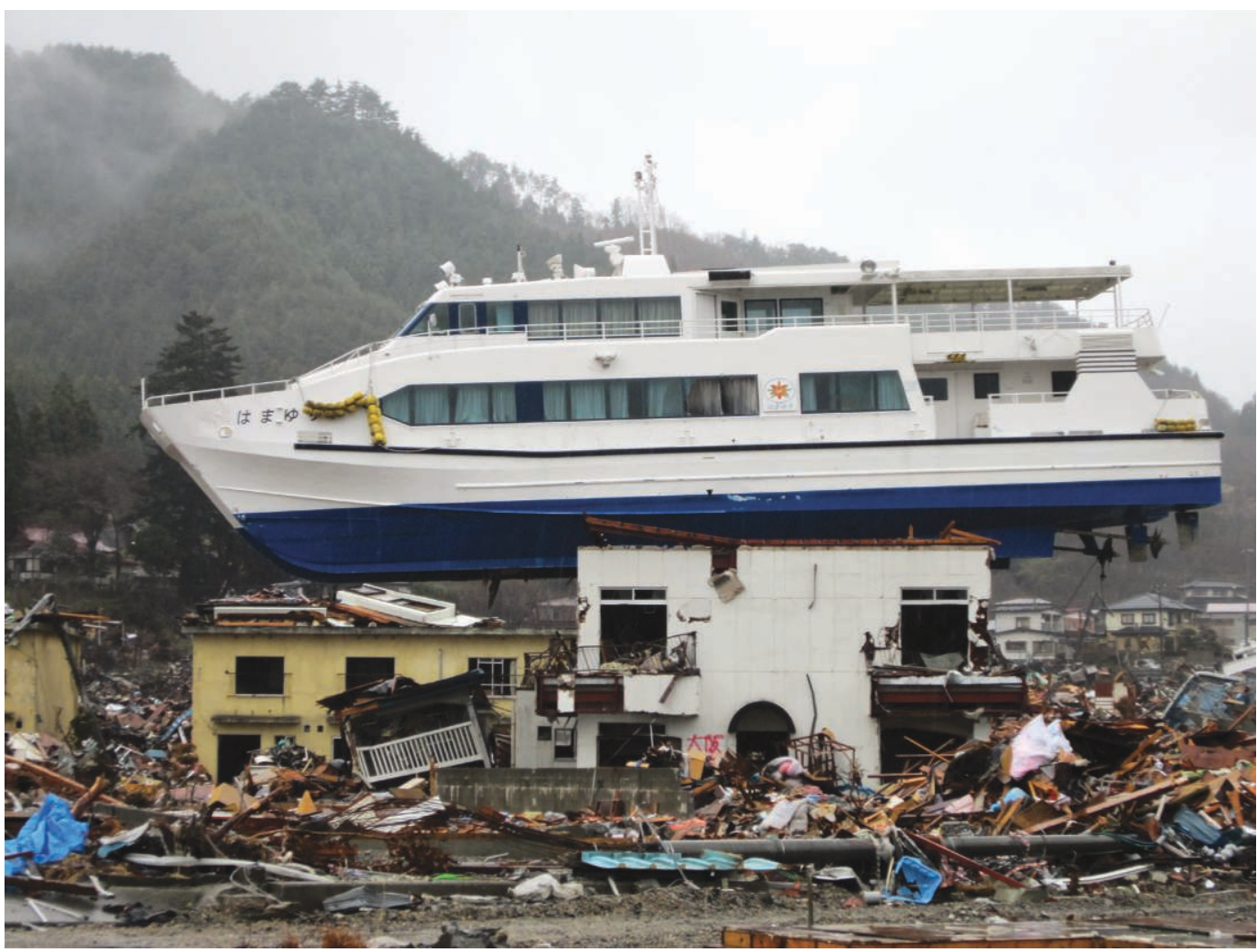

Pour quelle raison ces restes ont-ils été déjà démolis? Les maires qui ont décidé de leur démolition affirment que leur conservation pourrait offenser les proches des victimes et accroître leur sentiment de tristesse. Mais cette explication est inexacte, comme le montre l'exemple de la mairie d'Otsuchi. Pour discuter du projet de conservation des ruines de la mairie, le maire a formé une assemblée délibérante représentative, constituée de trois professeurs, deux proches des victimes (l'un pour et l'autre contre la conservation), deux élèves du lycée, deux membres du conseil municipal et un employé de la mairie. Cette assemblée s'est réunie et a délibéré pendant trois mois afin de présenter un rapport final indiquant les avantages et inconvénients de ce projet sans pour autant prendre de décision dans un sens ou dans un autre. À la suite de ce rapport, le maire a affirmé que la mairie devait être conservée, mais, en raison des avis très partagés, le conseil régional n’a pas encore approuvé cette décision. En effet, l'assemblée a fait une enquête auprès des 37 proches des victimes et 247 employés travaillant actuellement à la mairie. Environ la moitié des proches des victimes s'est prononcée en faveur de la conservation des restes du bâtiment existant et l'autre moitié, contre. En revanche, une majorité écrasante des employés de la mairie (79\%) ne souhaitent pas sa préservation. Nous n'avons pas encore réalisé d'enquête systématique chez les habitants de la ville, mais au cours de nos entretiens, nous avons pu constater la même tendance. 
Pour comprendre pourquoi beaucoup d'habitants se montraient hostiles à cette conservation et pour savoir ce que nous devions faire pour modifier leur point de vue, nous avons entamé des entretiens avec plusieurs proches des victimes. Parmi eux, il y avait une dame et un jeune homme qui étaient respectivement pour et contre le projet de sauvegarde de l'actuelle mairie. Lors du tsunami, la dame a perdu son mari et sa fille unique qui travaillait à ladite mairie. C'est une personne dynamique, gaie et bavarde; elle est la responsable de l'association des femmes de la ville. Néanmoins, elle nous a expliqué qu'elle n'a pas pu aller voir la mairie pendant un an et demi, l'idée de s'y rendre l'effrayait. Un jour, une de ses amies l'a amenée devant la mairie, et c'est à ce moment qu'elle a cru que sa fille perdue lui adressait la parole. Elle a compris alors que sa fille resterait là pour l'éternité et qu'elle devait tout faire pour conserver les restes de ce bâtiment. Depuis ce jour, elle a lancé une campagne en faveur de la conservation de la mairie, considérant qu'elle est le meilleur symbole pour transmettre aux générations futures l'étendue de la force destructrice du tsunami. Elle souhaite que les gens viennent voir cet édifice et que cela contribue à l'amélioration de la situation économique de la ville dont toutes les industries ont été détruites.

Nous avons fait un autre entretien avec un jeune homme qui a perdu ses parents et son frère aîné employé également à la mairie. Sa maison se trouvant à proximité, il voyait le bâtiment de la mairie tous les jours depuis son enfance. Son état actuel de destruction lui procure un sentiment de tristesse et d'impuissance. Il comprend la nécessité de construire quelque chose pour témoigner de l'effrayante force du tsunami. Cependant, il pense que d'autres monuments bien conçus et mieux construits seront plus efficaces que ce bâtiment misérablement délabré. Il voudrait qu'il soit démoli le plus tôt possible, car sa vue l'emplit à chaque fois d'un peu plus de peine et de découragement.

Concernant les opinions recueillies au cours de l'enquête réalisée par l'assemblée délibérante, différents arguments s'affrontent. Pour ceux qui sont en faveur de la conservation, les restes de ce bâtiment pourraient devenir le lieu par excellence de la protection de la mémoire des victimes du tsunami, un cri d'alarme témoignant de la force destructrice du tsunami ou un mémorial attractif pour les touristes et les étudiants en voyage scolaire. Pour ceux qui sont opposés à la conservation, si le bâtiment entretient la désolation et l'abattement des habitants, il risque d'attirer des touristes impolis et voyeurs qui provoqueront le chagrin des proches des défunts. Un monument plus beau et mieux bâti serait plus utile que l'édifice en ruine.

Les opinions qui approuvent la sauvegarde de la mairie me semblent très raisonnables et bien fondées. En fait, beaucoup de personnes étrangères à la ville et de chercheurs sont de cet avis. Mais ces arguments raisonnables ne suffisent pas à convaincre les habitants qui sont majoritairement contre ce projet, ces ruines ravivant un traumatisme toujours présent chez eux.

Nous devons donc être très sensibles aux sentiments profonds qu'éprouvent les habitants. Et si nous essayons de réaliser une exposition sur le tsunami, nous serons certainement confrontés à la même objection, en dépit de la bienveillance de notre acte. Il nous faut donc chercher un moyen d'apaiser les émotions traumatiques que les habitants ressentent encore à la vue de la mairie et ce, bien que 5 ans se soient écoulés depuis la destruction par le tsunami. Est-il possible pour nous d'atténuer ce sentiment traumatique d'une façon ou d'une autre? 


\section{La communauté qui peut se battre contre le trauma}

L'expérience au cours de notre séjour à Iwate est significative pour comprendre la réaction de la communauté locale face à la catastrophe.

Kirikiri est une commune où la notion de collectivité est extrêmement forte. Après le tsunami, cette commune a été complètement coupée du monde extérieur pendant une semaine. Les habitants ont dû œuvrer ensemble pour sauver la vie des survivants, pour ouvrir une voie et un héliport en débarrassant des gravats, ainsi que pour préparer la nourriture des 600 réfugiés. Dans le même temps, ils ont examiné les cadavres (100 ont été dénombrés) pour les identifier par eux-mêmes sans recours aux examens ADN.

Ils nous ont raconté combien cet examen était pénible, car les corps avaient été détériorés avec le temps ou par le fait que certains organes (nez et yeux) avaient été mangés par les poissons. Nous leur avons demandé s'ils ne revoyaient pas leur image, s'ils ne rêvaient pas d'eux. «Jamais », nous ont-ils répondus, et ils nous ont affirmé ne pas avoir ressenti d'expériences traumatiques. Et cela à la différence des policiers, dont $15 \%$ ont éprouvé un trauma après avoir travaillé dans la région dévastée par le tsunami. Quelles sont les raisons de cette différence? En regardant de près l'histoire des études sur le trauma, on constate que la compréhension de ce dernier a fait de grands progrès

6. Les habitants ont travaillé ensemble pour enlever les gravats et rechercher les cadavres qui se trouvaient enfouis.

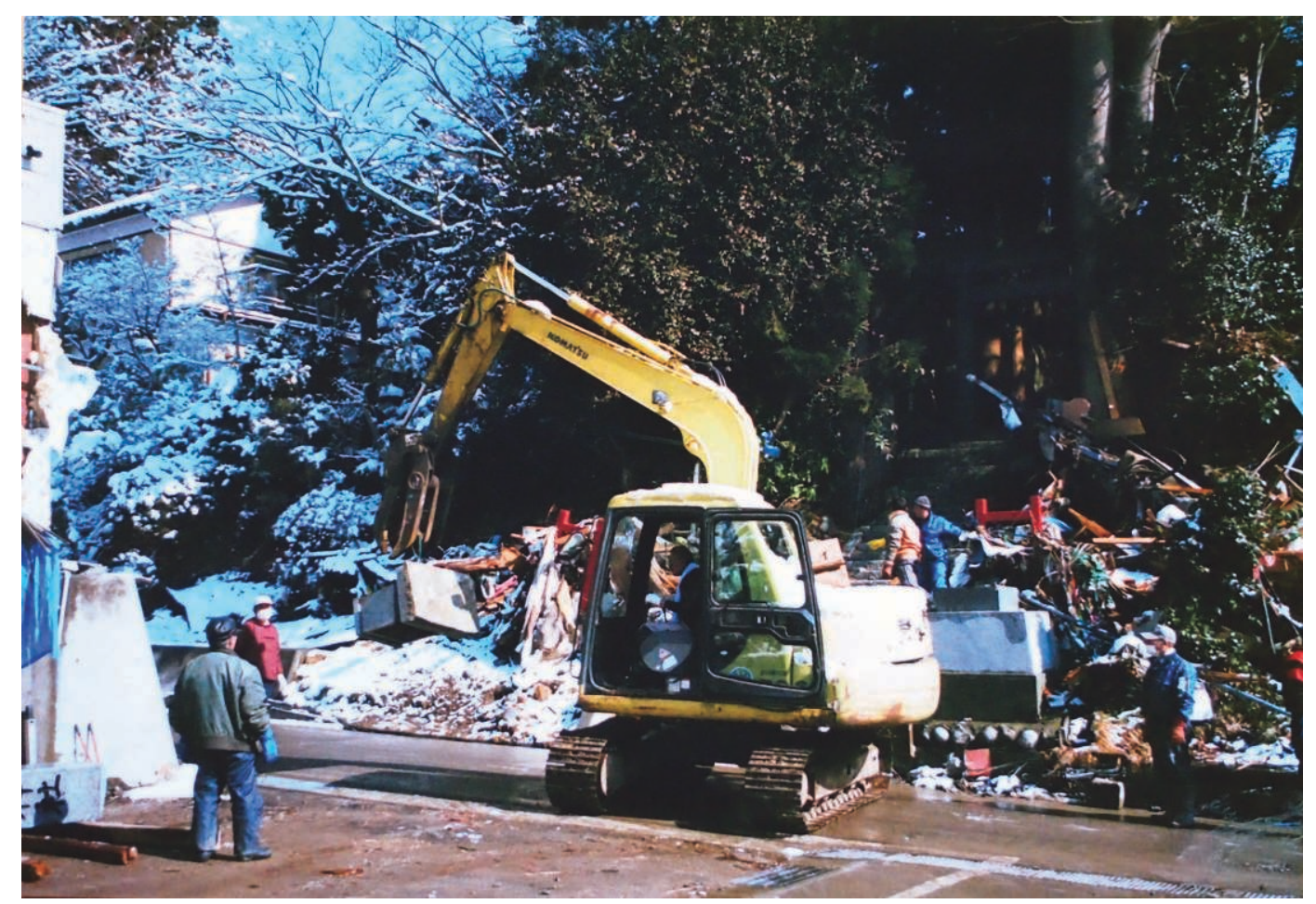


après la première guerre mondiale. En effet, les médecins ont remarqué à cette époque que beaucoup de soldats souffraient des souvenirs traumatiques des événements qui les avaient éprouvés au cours de la guerre. Après plusieurs tentatives, ils ont découvert que le meilleur traitement pour guérir les patients résidait dans la remémoration des liens sociaux qu'ils avaient pu nouer pendant la guerre, liens avec les autres soldats ou avec l'officier. Ce qui a abouti à la conclusion suivante: la solidarité est le meilleur remède d'apaisement du trauma (Herman 1992). Ces études sur l'histoire du trauma nous guident pour l'exposition que nous réalisons sur la catastrophe.

\section{Construire une exposition sur le tsunami}

Les cas cités nous semblent donc pertinents dans le cadre de la réalisation d'une exposition sur le tsunami. Si nous présentons une exposition focalisée sur la catastrophe, elle ne sera jamais acceptée par les habitants qui en souffrent encore dans leurs mémoires. Par contre, si nous pouvons construire une exposition qui leur rappelle la notion de solidarité, elle aura plus de chance d'être approuvée.

En quoi consisterait donc une exposition sur la solidarité? Pour l'exposition du Minpaku, à Osaka, nous avons cherché les photographies prises avant la catastrophe. Comme nous avions rassemblé de nombreux clichés de très bonne qualité, nous avons décidé d'organiser deux expositions qui ont eu plus de succès que nous n'avions imaginé (plus de 1000 visiteurs à chaque fois). De plus, beaucoup d'entre eux ont pleuré en regardant les images. Ils ont dit que ces photographies reproduisaient les scènes perdues, les scènes qui leur rappellent le meilleur moment de leur vie. Ils ont aussi affirmé que ces images incarnaient la transmission des mémoires communes dans lesquelles chaque mémoire personnelle se reflétait. Ces exemples nous ont ainsi suggéré la possibilité de la future exposition. Si nous recomposons attentivement et soigneusement les vies quotidiennes et religieuses des habitants avant le tsunami, l'exposition aura plus de chance d'être acceptée, voire d'être appréciée par les survivants.

Cette première partie sur la ville avant le tsunami sera suivie par une deuxième focalisée sur la catastrophe, qui sera constituée de photographies, de projections vidéo et d'exposition des restes. Cette mise en scène ne sera certainement pas appréciée par les locaux mais elle est indispensable pour attirer les intérêts des étrangers vers la ville. Et nous pensons qu'il serait inutile de réaliser une exposition qui n’attire pas les étrangers, car une exposition qui ne suscite pas le dialogue ne mérite pas d'être montée.

La scène finale exposera la vie post-tsunami: à partir de vidéos qui illustrent comment les habitants ont collaboré entre eux après le cataclysme et comment ils imaginent la ville reconstruite dans l'avenir.

Sur la photographie ci-contre, prise le lendemain de la catastrophe, nous remarquons une machine en mouvement et plusieurs hommes autour d'elle. Ce sont les habitants à la recherche des cadavres restés coincés sous les gravats. Et, comme la machine risquait d'abîmer les corps, ils devaient regarder très attentivement ce qui se trouvait sous les décombres. 
7. Une scène de festival d'Otsuchi juste après la deuxième guerre mondiale.

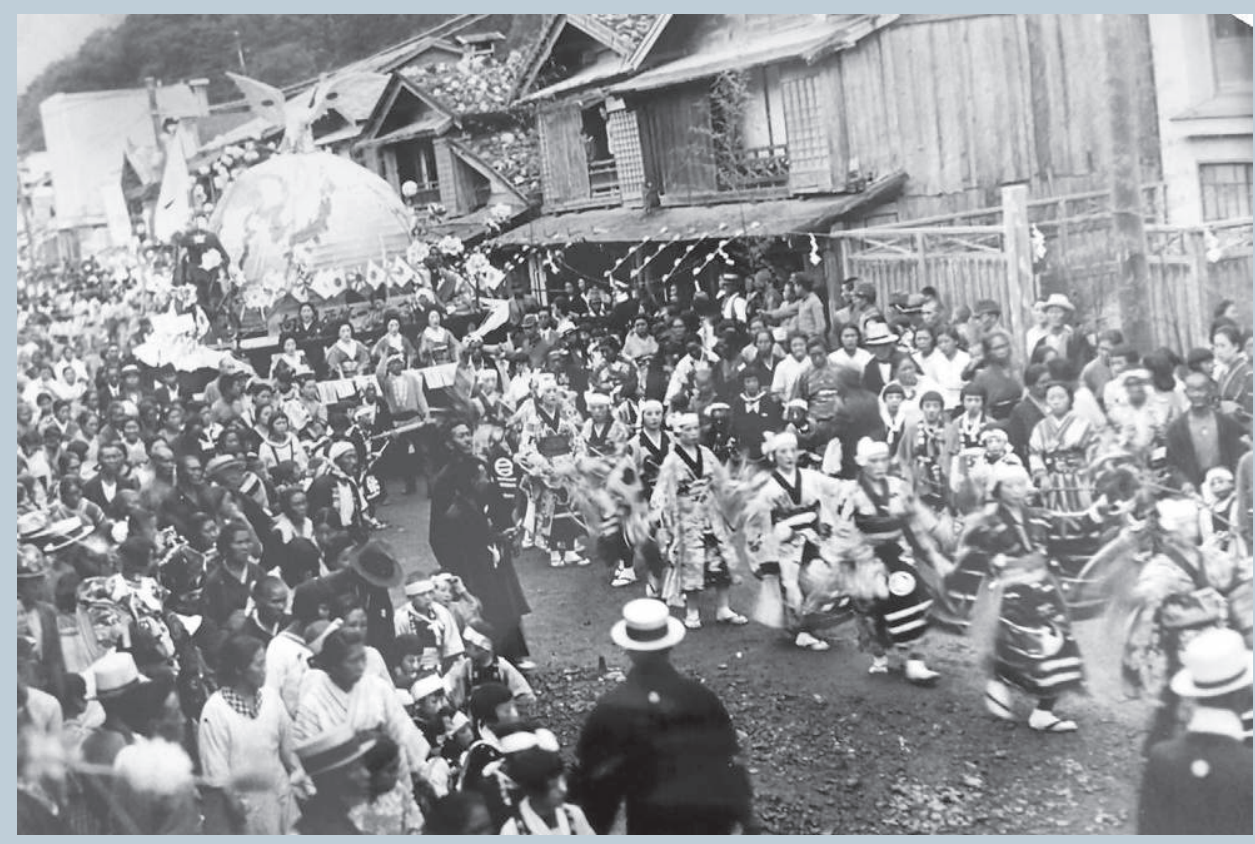

. La pêche du saumon a constitué une des principales ressources de la vie économique.

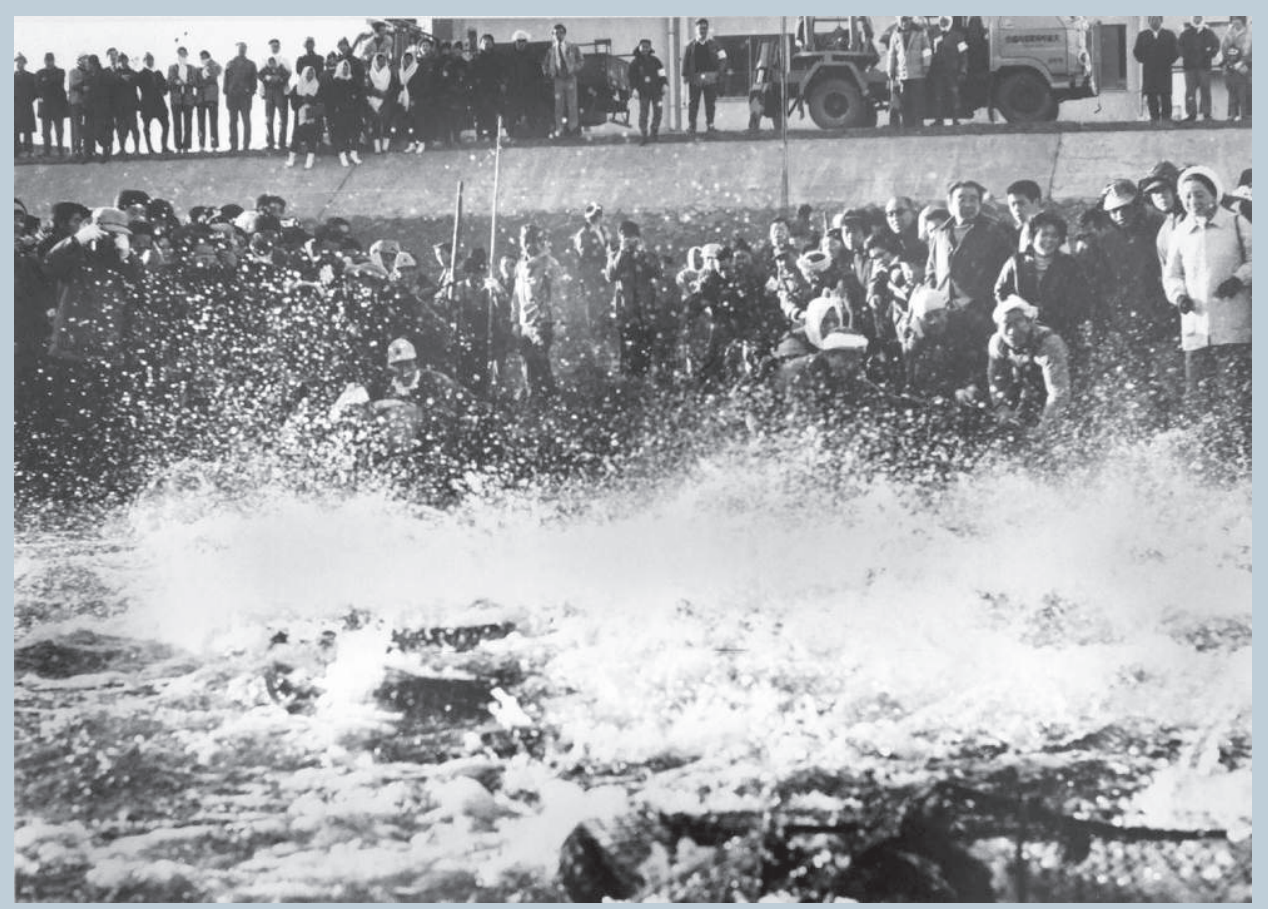


Ces scènes risquant de donner au visiteur une impression d'incohérence, nous souhaitons les structurer avec l'aide d'un habitant. Kemuyama Yutaka sera notre guide : il était commerçant de détail dans un des villages d'Otsuchi, mais il était surtout connu comme le chef de l'équipe des pompiers volontaires de l'ensemble de la ville dont quatorze parmi eux ont malheureusement trouvé la mort, en essayant de sauver les personnes âgées et malades. La mère, l'épouse et le fils unique de cet homme ont aussi été emportés par le tsunami. En raison de sa tristesse et de son sens de la responsabilité, il n’a jamais raconté son histoire en public ni accepté d'être interviewé. Un de ses amis nous a cependant indiqué qu'il aimait prendre des photographies et qu'il en possédait de très bonne qualité, et c'est ainsi que nous avons commencé à parler avec lui de la possibilité de présenter ses images. Comme elles étaient très bonnes et très élaborées, nous l'avons encouragé à organiser une exposition qui eut un grand succès. Il a alors recouvré de l'énergie et a recommencé à prendre des photographies et des vidéos pour enregistrer ce que devenait son village après la catastrophe. Nous le croyons très apte à représenter la ville car ses activités ont couvert beaucoup de domaines de la vie quotidienne de la ville d'Otsuchi, et nous avons construit l'exposition sur la base d'un scénario qu'il a inspiré: sa vie avant le tsunami, son expérience de la catastrophe, la manière dont il a survécu, et bien sûr comment il envisage le futur de la ville.

\section{L'auteur}

Shoichiro Takezawa est professeur au Musée national d'ethnologie, à Osaka (Japon). Ses publications principales comptent «La Fête japonaise. Appareil hégémonique de la cité marchande», Techniques\& Culture 57 (2011/2): 98-119; Sur la trace des grands Empires. Recherches archéologiques au Mali, numéro spécial des Études maliennes, 2016 (avec Mamadou Cissé); The Aftermath of the 2011 East Japan Earthquake and Tsunami, Lexington Books (sous presse).

\section{Iconographie}

Image d'ouverture. Le lendemain de la catastrophe, la ville a été totalement détruite par le tsunami et un grand incendie. 1 et 2. (C) Yoshiharu Ogawa.

3 à 5 . (C) Shoichiro Takezawa.

6. () Toshiaki Fujimoto.

7. (C) Masao Usuzawa.

\section{Références}

Herman, J. L. 1997 Trauma and Recovery. New York: Basic Books.

Takezawa, S. (à paraître en 2016) The Aftermath of the 2011 East Japan Earthquake and Tsunami. Lanham: Lexington Books.

\section{Pour citer cet article}

Takezawa, S. 2016 «Par-delà le trauma. 11 mars 2011, Iwate (Japon)», TechniquesECulture 65-66 « Réparer le monde. Excès, reste et innovation», p. 48-59. 